Polymer Journal, Vol. 2, No. 5, pp 672-674 (1971)

SHORT COMMUNICATION

\title{
Synthesis of Polyamide by Phosphoroxidation
}

\author{
Naoya Ogata and Hozumi Tanaka \\ Department of Chemistry, Sophia University, \\ Chiyoda-ku, Tokyo, Japan. \\ (Received April 16, 1971)
}

KEY WORDS Polyamide / Triphenyl phosphite / Imidazole / Phosphoroxidation /

It was found ${ }^{1}$ that a condensation reaction takes place at room temperature between $\alpha$ amino acids to form almost quantitatively a dimer in dimethylformamide in the presence of triphenyl phosphite and imidazole.

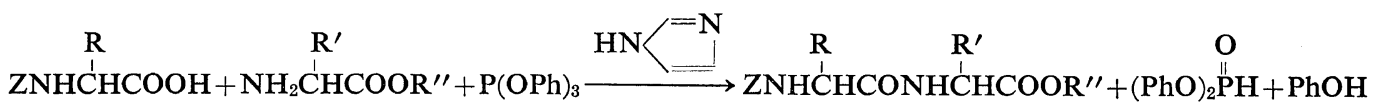

This reaction was extended to the polycondensation reaction of nylon salt or $\omega$-amino acids to obtain polyamides at room temperature.

A portion of $0.0025 \mathrm{~mol}$ of various nylon salts from diamines and dicarboxylic acids or 0.005 mol of $\omega$-amino acids was dissolved or suspended in $5 \mathrm{ml}$ of solvents, and $0.0076 \mathrm{~mol}$ of triphenyl phosphite and imidazole was added to this solution. The whole solution was kept at room temperature for a given period and stirred continuously.

The solution or suspension started to change gradually to a heterogeneous phase with the formation of a gel-like polymer. Polymers were collected by pouring the solution into acetone followed by filtration. Polymers were washed with water in order to remove unreacted monomers, and dried. The solution viscosity of the resultant polymers was determined in concentrated sulfuric acid at $30^{\circ} \mathrm{C}$.

Results of the polycondensation of hexamethylenediamine with adipic acid in dimethylformamide(DMF) are shown in Table I, where it is seen that polyamide was obtained quantitatively in DMF or DMAc, although the solution viscosity of the polymer was not high enough. The yielded polyamide was identified as nylon 66 from its melting point, elementary analysis, and infrared spectrum.
Table I. Polycondensation of hexamethylenediamine and adipic acid ${ }^{a}$

\begin{tabular}{lccc}
\hline \multicolumn{1}{c}{ Solvent } & Time, hr & Yield, $\%$ & $\eta_{\mathrm{sp}} / \mathrm{c}$ \\
\hline Dimethylformamide & 43 & 100 & 0.16 \\
Dimethylacetamide & 43 & 100 & 0.16 \\
Dimethylsulfoxide & 45 & 90 & 0.09 \\
$N$-Methylpyrrolidone & 42 & 85 & 0.19 \\
Triethylamine & 44 & 39 & 0.13 \\
Di-isobuthyl keton & 42 & 11 & 0.15 \\
Acetophenone & 42 & 17 & 0.09 \\
Methyl Acetophenone & 42 & 22 & 0.09 \\
Tetrahydrofuran & 43 & 81 & 0.17 \\
Dioxane & 42 & 81 & 0.14 \\
Benzene & 44 & 25 & 0.14 \\
$n$-Hexane & 44 & 33 & 0.10 \\
Ethanol & 43 & 0 & - \\
$m$-Cresol & 44 & 0 & - \\
Water & 22 & 0 & - \\
None & 45 & 0 & - \\
\hline
\end{tabular}

a Polycondensation was carried out at room temperature with $0.0025 \mathrm{~mol}$ of monomer in $5 \mathrm{ml}$ of solvents.

Since the apparent termination of the growth reaction in DMF or DMAc might be ascribed to the heterogeneous reaction phase with the precipitation of the polymer, lithium chloride was added to DMF in order to bring about the reaction in homogeneous phase. However, no 
effect of lithium chloride was observed and the amount of imidazole could be reduced by half to obtain polyamide almost quantitatively, as seen in Table II.

The polycondensation reactions of various

Table II. Polycondensation of hexamethylenediamine and adipic acid $^{a}$

\begin{tabular}{ccccc}
\hline $\begin{array}{c}\text { Imidazole, } \\
\text { mol }\end{array}$ & $\begin{array}{c}\text { LiCl } \\
\text { mol }\end{array}$ & $\begin{array}{c}\text { Time, } \\
\mathrm{hr}\end{array}$ & $\begin{array}{c}\text { Yield, } \\
\%\end{array}$ & $\eta_{\mathrm{sp}} / \boldsymbol{c}$ \\
\hline 0.0076 & 0 & 43 & 100 & 0.16 \\
0.0038 & 0 & 45 & 94 & 0.17 \\
0.0076 & 0.005 & 25 & 81 & 0.17 \\
\hline
\end{tabular}

a Polycondensation was carried out with 0.0025 mol of nylon salt in $5 \mathrm{ml}$ of DMF at room temperature. nylon salts or $\omega$-amino acids in DMF were carried out and results are summarized in Table III. Aliphatic nylon salts yielded polyamide quantitatively, while $\omega$-amino acids did not form polyamides at good yield.

The reaction mechanism of the polycondensation in the presence of triphenyl phosphite and imidazole is not yet clear. Gas chromatography analysis of the reaction solution identified the existence of amounts of phenol equal to that of the monomer, and the reaction of adipic acid with triphenyl phosphite in THF in the presence of imidazole yielded a crystalline product which was identified as a mixed anhydride containing the phosphite group.

Therefore, the reaction might proceed through the formation of a mixed phosphorous acid

Table III. Polycondensation of various monomers in $\mathrm{DMF}^{\mathrm{a}}$

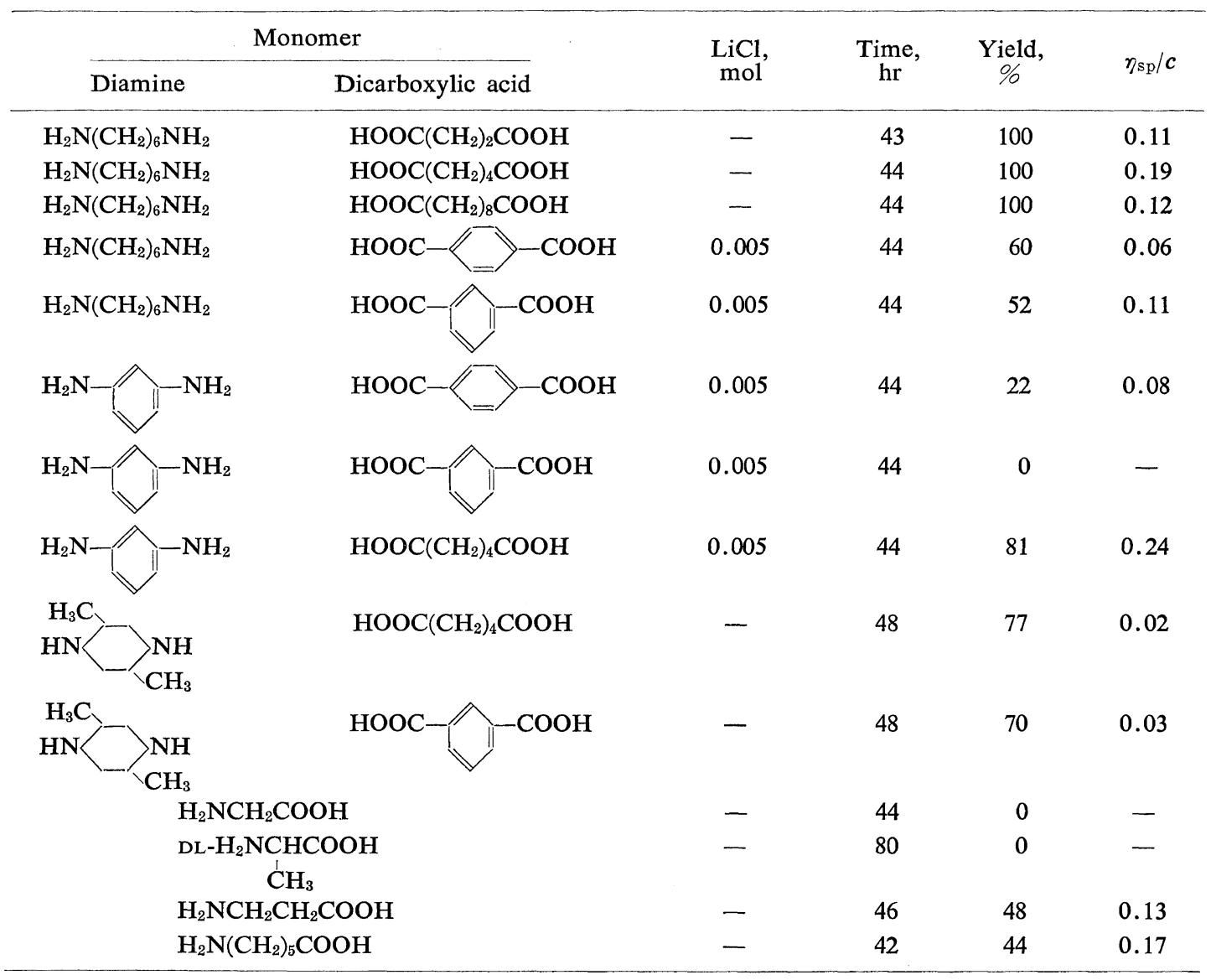

a Monomer concentration is $0.005 \mathrm{~mol} / \mathrm{in} 5 \mathrm{~m} l$ of DMF. 


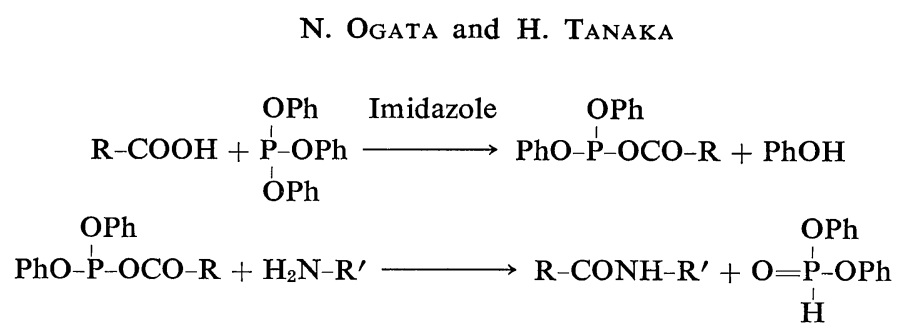

anhydride followed by aminolysis.

Further study of this polycondensation reac-

It is possible that imidazole plays a role as tion is in progress and the results will appear a catalyst or an acid acceptor for the formation of the mixed anhydride. Diphenyl phosphite might be transformed into penta-valent diphenyl phosphonate and this valency change might be the driving force behind this polycondensation reaction.

\section{REFERENCE}

1. Yu. V. Mitin, et al., Tetrahedrons Letters, 5267 (1969). 